\title{
Auf DER SCHWELLE VERHARREN. ZU EINEM ERzÄhlmuster der Moderne
}

\author{
RALF SIMON
}

\section{Die Gastlichkeit der Erzählung und die ungastliche Urbanität}

In alten Zeiten hatte man hohe Begriffe von den Rechten der Gastfreundschaft. Noch pflegen diese Begriffe in Ländern und Provinzen, die weniger bevölkert sind oder wo einfachere Sitten bei weniger Reichthum, Luxus und Corruption herrschen, so wie auf dem Lande, in Ausübung gebracht und die Rechte der Gastfreundschaft heilig gehalten zu werden. In unsern glänzenden Städten hingegen, wo nach und nach der Ton der feinen Lebensart allen Biedersinn zu verdrängen anfängt, da gehören die Gesetze der Gastfreundschaft nur zu den Höflichkeits-Regeln, die jeder nach seiner Lage und nach seinem Gefallen mehr oder weniger anerkennt und befolgt oder nicht. ${ }^{1}$

1788 erschien Knigges Über den Umgang mit Menschen, aus dessen Fassung letzter Hand das Zitat stammt, zum ersten Mal. Innerhalb einer möglichen Geschichte der Gastlichkeit wird hier eine Zäsur formuliert. Die Vorstellung eines einsam im Wald gelegenen Hauses, an das ein Fremder zu später Tageszeit anklopft und um Aufnahme bittet, die alternativenlose Notwendigkeit, ihn aufzunehmen, das Ausgeliefertsein des Gastes an den Wirt und die Gefahr des Wirtes, einen Unbekannten herein zu bitten: Diese Kernszene der Gastlichkeit zitiert eine Wirklichkeit, die im Europa des 18. Jahrhunderts schwindet. Die Infrastruktur des Reisens, die Siedlungsdichte, die Urbanisierung der Landschaft, die Technifizierung der Verkehrsmittel und die relative Risikolosigkeit einer weitgehend erforschten Welt hat die allgemeine Erfahrung der Gastlichkeit zunehmend aus dem kollektiven Bewusstsein getilgt. Knigges Buch über die Konventionen der Höflichkeit markiert diesen Einschnitt auf der Ebene des Diskurses. An die Stelle einer elementaren und archaischen Gastlichkeit wird die Konventionalität gesetzt. Heilig sind die Gastrechte nur noch in zurückgebliebenen Gegenden. Die moderne Urbanität aber folgt anderen Regeln.

1 Knigge: Umgang mit Menschen, S. 234. 
Wenn E.T.A. Hoffmann in seinen Erzählungen die Szene der Gastlichkeit in das Zentrum rückt ${ }^{2}$, dann wird man unterstellen müssen, dass diese umfangreiche Reflexion zwar noch mit der Erinnerung, aber auch schon mit dem Depotenziertsein der Gastlichkeit rechnet. Die Erzählliteratur des 19. Jahrhunderts mit ihren Zentren beim historischen Roman und beim anverwandelten Volksmärchen kennt viele Evokationen einer als ursprünglich imaginierten Szenographie des Gastes, aber es handelt sich wohl insgesamt um sentimentalische Rekonstruktionen. In dem Moment, in dem die Erzählung thematisch wie formell in der fortgeschrittenen Moderne angekommen ist, scheint ihr der Zugang zumindest zur Erfahrung der Gastlichkeit verwehrt zu sein. Wollte man diese Vermutung sowohl geschichtsphilosophisch als auch in der Terminologie der Gastsemantik formulieren, dann wäre insgesamt die moderne Erzählung so zu charakterisieren, dass sie in paradoxer Weise die Intention auf die Szene der Gastlichkeit beibehält, sie aber zugleich strukturell verhindert.

Die Erzählung lässt sich in der grundsätzlichsten Weise als ein Vermittlungsgeschehen bestimmen, welches zwei zueinander in Opposition stehende semantische Bereiche durch Aktantenbewegungen temporal vermittelt. ${ }^{3}$ Indem derart der Aktant der einen Semantik zum anderen Aktanten kommen muss, damit die Vermittlung erzählt werden kann, ist der Erzählung die Gastszene strukturell eingeschrieben. Immer ist einer beim anderen zu Gast. Vielleicht ist dies die in ihrer Kürze lakonischste Definition der Narration. Sofern die Erzählung auf der thematischen Ebene eine Handlung hat, die durch Aktanten ausagiert wird, muss einer zum anderen gehen. Das Ankommen des einen semantischen Eigenschaftsbündels unter den Bedingungen einer andersgearteten Semantik ist im Kern eine wie auch immer verwandelte Situation der Gastlichkeit. ${ }^{4}$ Deshalb kann die Erzählung als solche die Szenographie der Gastlichkeit nicht abschütteln, sie ist ihr strukturell eingeschrieben, sie bildet das ikonische Zentrum des Erzählfortganges, der offenkundig erst sekundär einem semantischen Prozess folgt. ${ }^{5}$

Die Erzählung ist nicht nur in ihrer inneren Struktur auf die Szenographie der Gastlichkeit angewiesen. Auch in der realen Gastlichkeit ist sie als eine wesentliche Position vorhanden. Denn der Gast bringt zunächst und elementar sich selbst: Er liefert sich dem Wirt aus. Vielleicht bringt er ein Gastgeschenk mit, um die schwierige Szene der Gastlichkeit durch ein Tauschgeschehen zu konventionalisieren. Er

2 Vgl. Simon: »Ungastlichkeit«.

3 Lotman: Struktur literarischer Texte, bes. Kap. VIII.

4 Um ein Missverständnis zu vermeiden: Der Begriff > Situation der Gastlichkeit $<$ ist rein formal verstanden (ebenso später: >Szene der Gastlichkeit<, >Gastsemantik< etc.) und präjudiziert nicht die gastliche Aufnahme. Gemeint ist, dass eine Situation etabliert ist, die den Anspruch der Gastlichkeit aufstellt, deren Einlösung aber nicht garantiert.

5 Vgl. Simon: »Ikononarratologie $«$. 
bringt aber auch die Erzählung mit. Sie ist seine primäre Gabe. Denn der Wirt wird den Gast um Herkunft, Reiseweg und Erfahrungen befragen und der Gast wird erzählen, was er in der Welt erlebt hat. Die Erzählung wiederholt in ihrer semiotischen Artikulation die Weltoffenheit, die mit dem Gesetz der Gastfreundschaft prinzipiell gegeben ist. So ist die Erzählung nicht allein in ihrem inneren Kern von der Gastsemantik geprägt, sondern in der realen Gastsituation selbst ein konstitutiver Faktor.

Eine einlässliche Theorie der Erzählung wird zu bedenken haben, wie diese doppelte Präsenz der Gastsemantik im Inneren der Narration als deren strukturelles Konstituens einerseits und als pragmatische Rahmenbedingung der Narration andererseits zu denken ist. Die Konstruktion einer genealogischen Vermutung liegt nahe und wird von der Struktur der Erzählung selbst nahegelegt: Es ist wohl die Hereinnahme der gastlichen Erzählszene in die innere Struktur dessen, was der Gast berichtet, welche allererst die Narration begründet. Der Gast berichtet dem Wirt, woher er kommt, was er erlebt hat, weshalb er reist und wohin er gehen wird. Dieses elementare Mitteilen findet im Rahmen der Gastlichkeitsszene statt. In dem Moment, in dem eine solche Situation wegfällt und sich die Erzählung als eigene Form aus dem Rahmen der Gastlichkeit löst, nimmt sie die vordem pragmatische Situation in sich hinein. Aus dem Bericht des Gastes über sein Herkommen und seine Absichten wird in dem Moment eine Erzählung, in dem das Erzählte seine Formbedingung in der Erzählung selbst aufrufen kann. Es wird also, aus genealogischer Perspektive gesprochen, die strukturelle Hereinnahme der Gast-Wirt-Konstellation ins Innere der Erzählung sein, die überhaupt erst aus einem Mitteilen oder einem Bericht die spezifische narrative Form erzeugt. So wird man die These, dass jede Erzählung notwendig das Ankommen einer semantischen Bestimmung bei einer anderen verhandelt, als das strukturelle Echo einer vormals konkreten Situation, in der Gast und Wirt sich austauschten, lesen können.

Methodologisch sei hier angemerkt, dass nicht freihändig behauptet werden soll, die Erzählung wäre in der Tat die Formalisierung dessen, was der Gast mitteilt - und es soll auch nicht unterstellt werden, dass derlei Grundsituationen dann immer noch das strukturierende Zentrum der Narration ausmachen würden. Der genealogischen Explikation ist deshalb eine zweite an die Seite zu stellen: Es ist der Konstruktivismus der Narration selbst, der diese Urszene entwirft und sie sich als seinen Grund zu- und einschreibt. Das $a$ versus $b$, das als semantische Opposition dem Erzählprozess zugrunde liegen soll, wird dann zu einem a geht $z u b$, wenn die semantischen Positionen zu Aktantenbewegungen werden. ${ }^{6}$ Wenn die Erzählung zwischen Anfang und Ende eine Vermittlung

$6 \mathrm{Zu}$ diesem Grundtheorem der strukturalistischen Narratologie ( $a$ versus $b$ ) und der Überführung in a geht zu $b$ vgl. die Ausführungen in Simon: »Ikononarratologie«. 
erzählt, so kann unter den Bedingungen personifizierten Agierens deren Mitte nichts anderes sein, als dass $a$ zu $b$ geht, und dort eine Situation der Gastlichkeit vorfindet. Die Urszene der Gastlichkeit ist in diesem Sinne ein notwendiges inneres Konstrukt der Erzähllogik, sie wird von der Erzählung selbst als deren Voraussetzung präsupponiert.

Das Zitat aus Knigges bekanntestem Buch gibt aber eine andere Diagnose - eine der historischen Zäsur, die der strukturellen Verankerung der Gastsemantik im Inneren der Erzählung widerspricht. Die moderne Erzählung hat geschichtsphilosophisch die Sphäre desjenigen Biedersinns, in dem die Gastrechte heilig waren (um mit Knigge zu sprechen) hinter sich gelassen, sie ist ihr zuerst im 19. Jahrhundert eine sentimentalische Erinnerung der historistischen Erzählweise geworden und im 20. Jahrhundert zur Gänze hinter neuen Thematiken und Techniken verschwunden.

In paradoxer Verflechtung scheint also die Erzählung eine strukturelle Angewiesenheit auf die Szene der Gastlichkeit zu haben, während sie mit ihrem Eintritt in die Moderne diese Angewiesenheit verleugnet. Diese Situation wirft die Frage auf, wie das Zugleich von struktureller Affirmation der Gastlichkeit und ihrer manifesten Verneinung zu denken ist. Kann der Gast zugleich bejaht und verneint werden? Wie ist das zu denken?

\section{Die Verneinung des Gastes?}

Ist es möglich, die Gastlichkeit zu verneinen und den Gast abzuweisen? Diese etwas uneinsichtige Frage öffnet bei genauerem Hinsehen den Abgrund einer begriffslogischen Paradoxie. Zunächst scheint es so, dass man jederzeit in der Lage wäre, Gastlichkeit zu verweigern und eine Person abzuweisen. Sie wäre dann kein Gast. Wenn man aber die Position des Gastes auf einer anderen Ebene als derjenigen einer nur empirischen Aushandlung verorten möchte, dann stellt sich die Frage, ob nicht ein abgewiesener Gast ein Gast bleibt - eben einer, der abgewiesen worden ist.

Bei der Gastlichkeit scheint es sich um eine Bedingung der Kultur überhaupt zu handeln, um ein naturrechtliches Fundamental. Gesellschaften würden den Wärmetod der inzestuösen Reproduktion sterben, wenn sie sich der Aufnahme Fremder verweigern würden. Offenkundig entwindet sich die Kultur ihrem Naturzusammenhang in dem Moment, in dem sie das Andere und das Fremde zulässt, ohne es mit sich identisch machen zu müssen. Genau in diesem Moment entsteht die Anerkennung des Nicht-Eigenen und damit ein Raum der Offenheit, der Deutung und des Diskurses. Das archaische Mythem, dass ein Gott in der Gestalt eines armen Wanderers anklopft und um Aufnahme bittet, lässt sich als metaphysische Gegenprobe auf den notwendigen Fortbe- 
stand der kulturerzeugenden Gastlichkeit verstehen. Bietet der Wirt dem Fremden Haus und Ehefrau als seinen eigenen temporären Besitz an, so steckt in dieser Geste die Erwartung, die gewährte Gastlichkeit möge durch den der Ehefrau beischlafenden Gott zur Zeugung eines Halbgottes führen. Der Amphitryon-Stoff versteckt diesen fundamentalen kulturbegründenden Handel hinter der komischen Maske des Lustspiels, aber die mythologische Substanz dieser Erzählung berichtet das Skandalon, dass der die Gastlichkeit prüfende Gott dem Wirt Haus und Ehefrau abverlangt. Der Ernst dieser Geschichte gibt Auskunft darüber, wie grundsätzlich und wie unverneinbar die Gastlichkeit ist. Denn erst, wenn dem Gott die Gastlichkeit gewährt wird, kann dieser den Fortbestand der Kultur garantieren und seinerseits einer Alkmene ein menschliches »Ach« angesichts metaphysisch induzierter Kulturgründung zugestehen.

Das Theorem, dass der Gast nicht verneint werden kann, impliziert also bei genauerem Hinschauen die schlechthin fundamentale Aussage, dass sich in der Gastlichkeit der generische Begriff der Kultur versteckt.

Versteht man nun also den Gast als denjenigen, der aus den genannten Gründen nicht verneint werden kann und der gleichzeitig, soll er Gast bleiben, nicht integriert wird - also weder $a$ noch non $a$, sondern vielmehr der Eintritt des ausgeschlossenen Dritten ist $^{7}$-, so stellt sich die Frage nach dem Status des abgewiesenen Gastes nunmehr in einer deutlicheren Form. Denn offenkundig ist es so, dass eine Person, der die Gastlichkeit verweigert wurde, deswegen keinesfalls aufhört, ein Gast zu sein. Sie wird vielmehr zu einem verneinten Gast. Da nun aber in der begrifflichen Konstruktionslogik des Gastbegriffes, weder $a$ noch non a zu sein, die Negation schon impliziert war, rückt die nochmalige Negation angesichts der Verneinung des Gastes in die seltsame Position einer Meta-Negation. Sie setzt sich gleichsam einem logischen Konstrukt auf, dem die Nichtverneinbarkeit schon inhärent ist, so dass die Verneinung nicht mehr negieren kann. Ist der Gast schon in der Ausgangsdefinition eine Figur des Dritten - das Gegebensein der Drittheit; tertium datur -, so scheint sich der abgewiesene Gast geradezu in eine logische Unmöglichkeit hinein zu bewegen.

Schon in der sozialen Erfahrung ist die Verweigerung der gastlichen Aufnahme eine verstörende und tief verletzende Erfahrung; sie geht weit über die Verweigerung der Anerkennung hinaus. Man muss einen Gast nicht anerkennen, um ihm Gastrecht zu gewähren. Das Gastrecht ist fundamentaler als die Anerkennung. Die Verweigerung des Gastrechtes beraubt den Gast selbst noch der Möglichkeit, derjenige zu sein, der nicht aufhört nicht zu kommen. Er wird zu einem, der beginnt, sein Nichtkommen zu sein. Im begriffslogischen Sinne verharrt er damit in

7 Bahr: Sprache des Gastes. 
einem Zwischenraum, den wir innerhalb der Szenographie der Gastlichkeit $^{8}$ als den Ort der Schwelle identifizieren können. Franz Kafkas Mann vom Lande, dessen Leben sich auf der Schwelle abspielt, so dass er weder gehen noch eintreten kann, aber auch nicht in der Lage ist, die Schwelle zu vermeiden, markiert in dem skizzierten begrifflichen Raum exakt die unmögliche Position des verneinten Gastes. Er verharrt auf der Schwelle, und die Erzählung, die dies zu berichten hat, wird darüber zu einer verharrenden Erzählung, zu einem narrativen Ritual der Wiederholung, zu einer oszillierenden Bewegung, die an einen Ort gebannt bleibt, der nicht einmal ein Nichtort ist: die Schwelle.

Zwei Gedankengänge liegen nun vor und führen zum selben Ergebnis. Sowohl die Verneinung des Gastes als auch die moderne Erzählung sind einer Logik überführt, die als Verharren auf der Schwelle charakterisierbar ist. Lässt sich diese Schwelle - die conditio narrationis der Moderne - als Bild der sowohl affirmierten als auch verhinderten Gastlichkeit näher bestimmen? Schwellenkunde ist ein schwieriges Terrain. Ich nähere mich ihm über einen Umweg, nämlich über den Nachweis, dass die Schwelle deshalb zum notwendigen Nichtort der Moderne wird, weil es dahinter nichts mehr gibt. In einer dekonstruktiven Umkehrfigur wird die Form der Verhinderung zur Erzeugung des Nichtseins dessen, was verhindert werden soll. Versuchen wir uns, dieser Schwelle zu nähern, indem zunächst zwei nichtnarrative Texte besprochen werden.

\section{Ein sentimentalisches Bedürfnis: Die erhoffte Reinheit der Gastlichkeit}

Obwohl es in diesem Beitrag um die Erzählung gehen soll, möchte ich meinen Ausgang bei zwei Gedichten nehmen. Beide Texte erhoffen sich eine Reinheit der Gastlichkeit, eine Erlösung von Schmerz und Fremdheit durch die Aufnahme. Somit formulieren beide Texte zu Beginn des 20. Jahrhunderts schon eine Utopie oder, wenn man so will, eine Wunschphantasie, von der sie wissen, dass sie nicht eintreten wird.

Ich weiss du trittst zu mir ins haus

Wie jemand der an leid gewöhnt

Nicht froh ist wo zu spiel und schmaus

Die saite zwischen säulen dröhnt.

Hier schreitet man nicht laut nicht oft .

Durchs fenster dringt der herbstgeruch

Hier wird ein trost dem der nicht hofft

Und bangem frager milder spruch.

Simon: »Ikononarratologie «. 
Beim eintritt leis ein händedruck ·

Beim weiterzug vom stillen heim

Ein kuss - und ein bescheidner schmuck

Als gastgeschenk: ein zarter reim. ${ }^{9}$

Das Gedicht Stefan Georges, das dem zweiten Teil aus Das Jahr der Seele entstammt, macht eine Abwehr des Unreinen zur Bedingung der imaginierten gastlichen Szene. Spiel und Schmaus, dröhnende Musik, der Ort der Vielen: Dies soll nicht die Situation der Gastlichkeit sein, im Gegenteil. In Georges Gedicht ist die begründende Szene, in der die sparsame Geste und das zarteste Geschenk die Aura der Eigentlichkeit erzeugt, erst durch die Negation einer uneigentlichen Realität zu erlangen. Bevor der Gast eintritt, müssen die Parasiten, die immer schon da sind, verdrängt und beseitigt werden: ins Nachbarhaus, zur Seite geschoben (para-sitos). Diese seltsame Geste der Abwehr, nach der Gastlichkeit nur sein kann, wenn vorgängig eine Purifizierung ungastlicher Bedingungen vorgenommen worden ist, markiert gegen die Intention des Gedichtes eine prosaische Politik, in der die prätendierte Eigentlichkeit das Ergebnis einer inszenatorischen Volte ist. Georges Gedicht legt via negationis davon Zeugnis ab, dass prosaische Zustände den $\mathrm{Zu}$ gang zu einer Sphäre lyrisch imaginierter Reinheit des Gastes und seiner Aufnahme blockieren. Die letzte Strophe scheint untergründig, eher in der Form als im ausgesagten Inhalt, die Gefährdung der Gastlichkeit zu formulieren. Händedruck und Kuss etablieren Bilder des Zusammenkommens zweier Personen über eine Differenz hinweg; und der Reim bestätigt genau dies, ist er doch die Vermittlung des Identischen im Verschiedenen. Das Gedicht beschreibt in der letzten Strophe nichts anderes als die Situation der Schwelle, denn der Druck der beiden Hände schwebt über der Grenze der Gastlichkeit, die die Schwelle ist, ebenso wie der Kuss. In dem Aufeinandertreffen der Lippen sind die beiden Personen, die diesseits und jenseits der Schwelle stehen, analog zum Reim gleichermaßen identisch gesetzt als auch in der Differenz gehalten. Liest man das Gedicht genau, dann wird man feststellen, dass die Szene der Gastlichkeit in ihm ausgespart ist. Vielmehr wird die Abwehr der ungastlichen prosaischen Zustände und die Situation der Schwelle als solcher zum Ort des Gedichtes - womit die Gastlichkeit, die es verspricht, zugleich auch revoziert wird.

Eine durchaus analoge Erfahrung findet sich etwa zeitgleich in einem Gedicht von Georg Trakl.

9 George: Das Jahr der Seele, S. 159. 
Ein Winterabend

Wenn der Schnee ans Fenster fällt,

Lang die Abendglocke läutet,

Vielen ist der Weg bereitet

Und das Haus ist wohlbestellt.

Mancher auf der Wanderschaft

Kommt ans Tor auf dunklen Pfaden.

Golden blüht der Baum der Gnaden

Aus der Erde kühlem Saft.

Wanderer tritt still herein;

Schmerz versteinerte die Schwelle.

Da erglänzt in reiner Helle

Auf dem Tische Brot und Wein. ${ }^{10}$

Im Wunsch nach der reinen Helle, die sich der Überschreitung der Schwelle und jenseits des Schmerzes zeigt, vibriert wie in jedem Gedicht von Trakl die Erfahrung einer inzestuösen Verunreinigung, einer prinzipiellen Vermischung der Kategorien, einer Spaltung jeder Position in ihre internen Ambivalenzen. Trakls Lyrik kontraimaginiert das Reine. Die Gastlichkeit als eine ursprünglich begründende Szene wird hier als starke Position formuliert, um den dunklen Pfaden und der schmerzversteinerten Schwelle den Ort einer nur sekundären Negativität zuzuweisen, der gegenüber Brot und Wein, die Erneuerung des Leibes, primär sein sollen. In überraschender Analogie zu Stefan Georges Gedicht wird auch hier die Gastlichkeit als ein utopischer Ort verstanden, dem der Schmerz, die Dunkelheit, die Versteinerung und das Unbehaustsein voran gehen. Einem genauen Blick zeigt sich jedoch, dass die Negation des gastlichen Hoffnungsbildes weitaus radikaler ist. Die Hoffnung, dass dem Gast mit Brot und Wein die Transsubstantiation seines Schmerzes werde, formuliert eine Unmöglichkeit. Der winterlichen Erde wird nichts erblühen können, und würde etwas blühen, so wäre eine goldene Blüte kein Ausweis eines rettenden Lebens, sondern vielmehr eine andere Form der Erstarrung, eine Mineralisation. Dass der Baum der Gnaden trotz Winter blühen könne und dass er dies golden statt grün tun solle: Die Impossibilia motivieren sich im Gedicht als unmögliches Hoffnungsbild aus dem Erglänzen in reiner Helle, welches Brot und Wein umgeben soll. Es handelt sich hier nicht um eine Gabe des Lebens, sondern um eine Verklärung, die den Tod zur Voraussetzung hat. Nichts anderes meint die Versteinerung der Schwelle. So zeigt sich, dass das Gedicht die Ankunft in der Gastlichkeit dem Jen-

10 Trakl, aus der Sammlung Sebastian im Traum (1915), also die zweite Fassung des Gedichts, in Trakl: Werk, S. 58. 
seits überlässt und dass die anfängliche Hoffnung, es werde einem Wanderer schon im Diesseits ein Ort bereitet, vergebens ist. Liest man das Gedicht in dieser Weise, dann verharrt es als solches auf der Schwelle. Es ist ein Gedicht, das den Übergang zum Tod ins Bild setzt und also die eine große und wesentliche Schwelle zu seinem Thema hat.

Beide Gedichte zeigen, dass jeweils die Szenographie der Gastlichkeit unterwandert und negiert wird. In Reinheit kann sie nur aufgerufen werden, wenn die Möglichkeit ihrer konkreten Realisierung dementiert wird oder wenn zunächst die prosaische Einrichtung der Welt einer Negation unterliegt. Man kann beide Gedichte als Untermauerung und Bestätigung der These lesen, dass die Gastsemantik in der Moderne ihrer konkreten Möglichkeit verlustig gegangen ist. Es gibt in der mitteleuropäischen Urbanität seit dem 18. Jahrhundert keinen Weg mehr in die Erfahrung der Gastlichkeit in Hinsicht jener Kernszene der Aufnahme des Fremden an der nächtlichen Schwelle.

Und so verharrt auch die Erzählung der Moderne auf der Schwelle zur sentimentalisch intendierten Kernszene. In diesem Sinne ist von einem Erzählmodell des an der Schwelle verharrenden Erzählens zu sprechen.

\section{Das verharrende Erzählen}

Die moderne Erzählung verharrt auf der Schwelle. Sie kommt nicht mehr im Inneren der Gastlichkeit an. Ihr wird der Eingang in das gastliche Haus und die Aufnahme als Gast verweigert, ohne dass sie aufhörte, Gast sein zu wollen und sein zu müssen. So verbleibt sie vor dem Gesetz. Kafkas Erzählen lässt sich in diesem Sinne als der Versuch verstehen, den Ort der Schwelle zu einem ganzen labyrinthischen System auszubauen. Die Schwelle wird ihm zum >Bau< ${ }^{11}$, thematisch wie erzähltechnisch. Kafkas Anverwandlung der Fabeln und überhaupt seine gesamte Welt der Supplemente, Ersetzungen, Verdopplungen und Verkehrungen ruht einer parasitären Semantik auf, welche den Eintritt in die Gastlichkeit verhindert und sich mit der vergeblichen Hermeneutik

11 Kafkas 1923-1924 entstandene Erzählung Der Bau wird gerne als symptomatischer Text für die gesamte Text- und Weltkonstruktion Kafkas gelesen. Die unausgesetzte Absicherung, Verschiebung, Verdopplung und das Hineinkopieren der Grenze zwischen Bau und Außenwelt führt dazu, dass die Schwelle zu einem Ort der Permanenz wird. Ähnlich verhält es sich mit Josefine, die Sängerin oder das Volk der Mäuse: Was Josefine sei und ob sie überhaupt eine Sängerin ist, bleibt als Verhandlungsgegenstand des Textes in der Schwebe. Die Schwelle zur Bestimmung wird nicht überschritten. Es wird nichts erzählt. - In diesem Sinn lässt sich der Terminus des Baus hier bestimmt als Bau der Schwellensituation - als die thematische Kernszene Kafkas beschreiben. 
der Schwelle begnügt. Sie platziert sich vor oder neben der gastlichen Szene: parasitos.

Auch in Robert Musils Mann ohne Eigenschaften verharrt das Erzählen im Raum des Möglichkeitssinns. Der Roman verweigert den Eintritt in den Ort des Angenommenseins und verbleibt vor dem Wirklichen, schreckt davor zurück, sich dem Gesetz und seinen Anforderungen zu konfrontieren. »Seinesgleichen geschieht « ist bei Musil ${ }^{12}$ ähnlich wie Kafkas »stehender Sturmlauf ${ }^{13}$ die Formel eines Erzählens, welchem die Möglichkeit abhanden gekommen ist, eine reale Aufnahme des Aktanten und seiner Erzählung zu gewährleisten. Der Gegenpol zu dem »Seinesgleichen geschieht« ist im Roman die Liebe von Agathe und Ulrich, die als Inzest strukturell die Negation der Gastlichkeit vollzieht. Im Inzest kommt man immer nur im Eigenen an, ohne je angekommen zu sein. Man schließt den Fremden, den Gast aus. »Seinesgleichen geschieht « und Inzest: diese beiden Pole, die keine sind, weil sie sich in sich selbst auflösen, lassen sich im Kontext der hier geführten Argumentation als eine präzise Gegenthese zur Gastlichkeit lesen. Der Roman extendiert seine Schwelle. Trotz seiner Länge teilt er mit Kafkas Vor dem Gesetz dieselbe Grundidee: Das Erzählen verliert seinen inneren Motor, es geht ihm jener Ereignischarakter verloren, der in der Bitte um gastliche Aufnahme der einen Semantik bei der anderen bestand. Man kann vielleicht auch formulieren, dass ein Erzählen, das ins Geschwätz extendiert oder in den Inzest zusammenfällt, mit dem Verlust der erzählerischen Energie schlichtweg zur Prosa wird. In diesem Sinne wäre die Formel vom >Auf der Schwelle Verharren< vielleicht sogar nicht einmal der Name eines Erzählmusters, als vielmehr der Hinweis auf die Notwendigkeit einer Theorie der Prosa.

Man kann in diesem Sinne eine Vielzahl von modernen Texten in das angedeutete Exegeseschema einlesen. Ist nicht Thomas Manns Beschreibungskunst in den Buddenbrooks eine Suspendierung des Erzäh-

12 Die Formel des »Seinesgleichen geschieht « ist in Musils Roman bekanntlich die Überschrift des zweiten Teils, aber zugleich auch die Systemformel für eine leerlaufende Konversationskultur, die sich in ihren kommunikativen Ritualen einschließt und als eine Form der Ungastlichkeit gelesen werden kann. Wenn das >Seinesgleichen< aber vor allem auf die Eigenart des Verlaufs der Weltgeschichte, sich zu verlaufen, angewandt wird und >Geschichte< als Ergebnis ungesteuerter Konvergenzen kleiner Ursachen gedeutet wird, dann stellt Musil in dieser Reflexion des Geschichtsbegriffs zugleich denjenigen Ereignisbegriff in Frage, der nach Lotman (s.o.) für die Narration konstitutiv ist (vgl. Musil: Mann ohne Eigenschaften, S. 360f.). Die Diagnose, dass das Erzählen bei Musil in die Prosa, den Essayismus mündet und dergestalt narrativ auf der Schwelle verharrt, lässt den Mann ohne Eigenschaften als eine Variante des Verharrens auf der Schwelle erscheinen.

13 Vgl. Kafkas Tagebucheintragung des »stehenden Sturmlaufes« (20.11.1911; Kafka: Tagebücher, S. 259f.), der in der Variante des »stehendes Marschieren[s]« (23.01.1922, Kafka: Tagebücher, S. 887) später wieder aufgenommen wird. 
lens, dem gleichzeitig eine Kreisbewegung korrespondiert, in der die degenerative Wiederholung des krankhaften Schwächerwerdens alle Möglichkeit einer Aufnahme des Gastes negiert? Hier ist die Krankheit der Kultur in die Familie eingedrungen, so dass sich die Frage nach dem Gast gar nicht mehr stellt; entsprechend läuft die Erzählung in die Wiederholung oder in die Beschreibung aus. Ein anderes Beschreibungsexperiment findet sich in Peter Weiss' Der Schatten des Körpers des Kutschers, in dessen Zentrum eine Szene des gemeinschaftlichen Mahls steht. Diese simple Handlungssequenz, die in einem Satz zusammenzufassen wäre, nimmt bei Weiss mehrere Seiten ein. Die Beschreibung zerlegt die Szene in minimale Bewegungseinheiten. Darüber wird der Text so langsam, dass die Szene selbst in eine Art von Auflösung gerät. Gastlichkeit ist strukturell nicht mehr möglich, wenn allein das Heben des Essbestecks eine Seite in Anspruch nimmt. Nie wird ein Erzählereignis in einen Raum eintreten, in dem die Narration in die zergliedernde Zeitlupe der Prosa gestürzt ist. So langsam, wie hier die Prosa fortschreitet, kann kein Gast mehr eintreten.

Zum Formenrepertoire des Verharrens auf der Schwelle wird man den inneren Monolog zählen müssen. Joyce' Ulysses lässt den modernen Odysseus nicht ankommen, vielmehr wird er in eine Form überführt, die als literarische Verfahrensweise das verharrende Erzählen abbildet. Der innere Monolog schließt das Subjekt in seiner Schwellensituation ein und verhindert, dass die Rede an einem Ort aufgenommen würde. Formtheoretisch ist der innere Monolog das Verharren auf der Schwelle, die semiotische Permanenz eines Begehrens, welches im vormodernen Erzählen jenseits der Schwelle an den gedeckten Tisch gelangen wollte, um dort eine Erzählung zu platzieren, die ihren >Sitz im Leben< hatte. Aber Joyce' Roman endet in der Permanenz der Rede selbst.

In der deutschen Literatur ist vielleicht Hermann Brochs Der Tod des Vergil die konsequenteste Umsetzung des inneren Monologs. Die Sterbesequenz des Dichters wird zum extendierten Moment eines den ganzen Roman umfassenden inneren Monologs gemacht, so dass sich Brochs Roman geradezu als der paradigmatische Roman des verharrenden Erzählens bezeichnen lässt. Er hat in dieser Charakterisierung eine entschiedene Ähnlichkeit zu Hans Henny Jahnns Fluß ohne Ufer, dessen großräumige epische Wiederholungsstruktur den Handlungsfortgang zersetzt, um zu einer erzählerischen Retardation zu kommen, deren Ziel in der Reflexion der Schwelle zwischen Leben und Tod besteht.

Offenkundig scheint das Verharren auf der Schwelle in der Erzählliteratur der Moderne entweder zur miniaturisierten Form - Robert Walser, Kafka - oder zur grossen Form zu führen. Der stehende Sturmlauf der modernen Prosa zersplittert in seine nach innen gespaltenen Einheiten oder er zieht die ganze Enzyklopädie in seine Kreis- 
bewegung hinein. Beide Optionen verhindern das Erzählen und den Gast; sie gewinnen die Prosa und Selbstreflexion.

Denkt man an die fortgeschrittene Moderne, etwa an Arno Schmidts Bücher der 50er und 60er Jahre, so findet man dort Figurenentwürfe, in denen nach dem Kollaps des Realen ein rein virtueller Ort geöffnet wird, an dem die Gastlichkeit nur noch die Form einer Aufnahme in die Welt der Lektüren findet. Schmidts Protagonisten glauben nicht mehr an einen weltlichen Ort oder an die Möglichkeit einer gastlichen Aufnahme. Ihre Fluchtphantasien kulminieren in der Idee eines entleerten Raums, in dem sie sich nur noch in der Gesellschaft von Büchern befinden. Gastlichkeit wäre, wenn ein Buch sich öffnete und den Leser in sich aufnähme. Schmidt versucht also eine Umkehrfigur zu denken: Es ist nicht mehr die Gastlichkeit, welche die Erzählung leitet und in Szene setzt, sondern vielmehr ist der Raum der Texte derjenige, in den aufgenommen zu werden sich die Akteure von Schmidts Texten bemühen. Statt Gastlichkeit zu erzählen, soll die Literatur Gastlichkeit stiften. Die im 19. Jahrhundert nur noch sentimentalisch imaginierte Möglichkeit einer Erfahrung der Gastlichkeit ist hier nicht nur zum Diskurs geworden, vielmehr ist dieser Diskurs durchaus emphatisch an die Stelle der Möglichkeit der Erfahrung gerückt. Zettels Traum diskutiert hinter der Maske des Übersetzungsproblems genau dies: Welche Gastlichkeit kann die Welt der Bücher eröffnen?

Insgesamt kann man sagen - und das ist in der Tat nicht neu -, dass das moderne Erzählen keinen legitimen Ort mehr hat ${ }^{14}$, in die transzendentale Heimatlosigkeit ${ }^{15}$ entlassen ist und sich also in einer Bewegung befindet, welche ihre nun unmögliche Zentrierung in der Szene der Gastlichkeit gehabt haben soll, jedoch aus dieser grundsätzlich in die »tiefe Ratlosigkeit des Lebenden ${ }^{16}$ verstoßen ist. Das Erzählen ist keinem Wirt zugesprochen und es ist nicht mehr die Gabe des Gastes, die dankbar entrichtet wird.

Man kann die Frage stellen, ab wann sich die Erzählung ihre geschichtsphilosophische Signatur des auf der Schwelle Verharrens zugeschrieben hat. Vielleicht ist der früher einmal sogenannte >Bildungsroman< der letzte Versuch einer Leugnung der Tatsache, dass erzählerisch über die Schwelle nicht hinauszukommen sei. >Bildung<stand für den Versuch, einen gastlichen Ort wenigstens im Diskurs zu finden. Aber diese Bemerkung zieht das Recht ihrer Pauschalisierung aus einer allzu großen Entfernung zu den Texten, deren genaue Analyse jeweils nachzuweisen hätte, dass kein sogenannter >Bildungsroman $<$ jemals in

14 Vgl. Benjamins Aufsatz »Der Erzähler« (Benjamin: Gesammelte Schriften, Bd. II.2, S. 438-465).

15 Lukács: Theorie des Romans, S. 52.

16 Benjamin: Gesammelte Schriften, Bd. II.2, S. 443. 
der Lage gewesen ist, dem Helden eine Heimstatt oder auch nur ein Gastrecht zu gewähren.

Die Erzählungen des 19. Jahrhunderts haben in der deutschen Tradition zunächst ihre Modelle im Märchen und in der historischen Erzählung gesucht, also in den Imaginationen vergangener Lebenswelten. So konnte sich die Erzählliteratur recht lange um diejenige Konfrontation drücken, die das Verschwinden der Gastlichkeit in allen ihren Auswirkungen für das Erzählen zu gewärtigen gehabt hätte. Schon E.T.A. Hoffmann hat, indem er die Szenographie der Gastlichkeit rekapituliert und etwa im Ignaz Denner die komplette Kernszene auferstehen lässt, de facto eine ganze Enzyklopädie der Ungastlichkeit ${ }^{17}$ entworfen und darin gezeigt, dass der Erzählung das Haus verschlossen bleibt. Wilhelm Raabe lässt im späteren 19. Jahrhundert Figuren auftreten, denen wortwörtlich der Ort entzogen wird, an welchem die Bestimmung der Gastlichkeit einen Sinn gehabt hätte. Insgesamt unternimmt der poetische Realismus ${ }^{18}$ den Versuch, die Gastsemantik mit ihrer Kernszene ein letztes Mal zu bedenken, in der Form einer konkreten Verabschiedung: Raabe ist dabei der vielleicht expliziteste Autor, aber es lassen sich bei Stifter, Storm und Keller analoge Strukturen finden.

Nach dem realistischen Erzählen, das die Gastszene evoziert, um ihre geschwundene Geltung zu konstatieren, geht der Narration diese Szene verloren; sie wird zum auf der Schwelle verharrenden Erzählen. ${ }^{19}$ Weil die Erzählung weder einen Ort hat, an dem sie zu Gast ist, noch ihre interne Szenographie der Gastlichkeit ausformulieren kann, wird sie, ohne als Erzählung auf die Gastlichkeit verzichten zu können, selbst zum verneinten Gast, zur Prosa.

17 Vgl. Simon: »Ungastlichkeit«.

18 Vgl. Bürner-Kotzam: Vertraute Gäste.

19 Einer weiterführenden Debatte müsste die Frage unterbreitet werden, ob die HotelLiteratur des 20. Jahrhunderts und die Exil-Literatur neue Szenarien der Gastlichkeit entstehen lassen. Zu den Exiltexten stellt Rolf Parr in diesem Band die These auf, dass der Exilant weder die Gastlichkeit begehrt noch sie erhält, sondern die Heimkehr erwünscht, die sich dann als problematische zeigt. Die Hotel-Literatur entwirft die Szene einer professionellen und konventionalisierten Gastlichkeit, deren Anschluss an die Grundsituation der Erzählung nicht sogleich evident ist. 


\section{Literatur}

\section{PRIMÄRLITERATUR}

George, Stefan: Das Jahr der Seele (1897). In: ders.: Werke. Ausgabe in vier Bänden. München: dtv 1983.

Kafka, Franz: Tagebücher. Frankfurt am Main 2002 / Lizenzausgabe Darmstadt o.J. (Kafka, Franz: Schriften. Tagebücher. Kritische Ausgabe. Hrsg. von Jürgen Born, Gerhard Neumann, Malcolm Pasley und Jost Schillemeit).

Musil, Robert: Der Mann ohne Eigenschaften. 2 Bd. Reinbeck bei Hamburg: Rowohlt 1978.

Trakl, Georg: Das dichterische Werk. München: dtv 1980.

\section{SEKUNDÄRLITERATUR}

Bahr, Hans-Dieter: Die Sprache des Gastes. Eine Metaethik. Leipzig: Reclam 1994.

Benjamin, Walter: Gesammelte Schriften. Frankfurt am Main: Suhrkamp 1980.

Bürner-Kotzam, Renate: Vertraute Gäste. Befremdende Begegnungen in Texten des bürgerlichen Realismus. Heidelberg: Winter 2001.

Knigge, Adolph Freiherr: Über den Umgang mit Menschen. In: ders.: Ausgewählte Werke. Bd. 6. Hannover: Fackelträger-Verlag 1993.

Lotman, Jurij: Die Struktur literarischer Texte. München: Wilhelm Fink ${ }^{2} 1981$.

Lukács, Georg: Die Theorie des Romans. Darmstadt; Neuwied: Luchterhand 1977.

Simon, Ralf: »Die Nacht des Gastes. Zur Semantik der Ungastlichkeit in E.T.A. Hoffmanns >Nachtstücken««. In: Peter Friedrich und Rolf Parr (Hg.): Gastlichkeit. Erkundungen einer Schwellensituation. Heidelberg: Synchron 2009, S. 263-280.

Simon, Ralf: »Ikononarratologie. Bildtheoretische Grundlegung der Narratologie in der Szenographie der Gastlichkeit«. In: Alexander Honold und Ralf Simon (Hg.): Das erzählte und das erzählende Bild. München: Wilhelm Fink 2010, S. 303-329. 